\title{
Novel directions for psychiatric diagnosis: from psychopathology to motor function to monitoring technology
}

\author{
P. R. Bakker ${ }^{1,2}$, M. Wichers ${ }^{2}$, P. N. van Harten ${ }^{1,2}$, I. Myin-Germeys ${ }^{2}$, P. Delespaul ${ }^{2}$ and J. van Os ${ }^{2,3 *}$ \\ ${ }^{1}$ Psychiatric Centre GGZ Centraal, Amersfoort, The Netherlands \\ 2 Department of Psychiatry and Psychology, School of Mental Health and Neuroscience, Maastricht University Medical Centre, Maastricht, \\ The Netherlands \\ ${ }^{3}$ King's College London, King's Health Partners, Department of Psychosis Studies, Institute of Psychiatry, London, UK
}

\begin{abstract}
In the light of the recent publication of the DSM-5, there is renewed debate about the relative merit of categorical diagnosis, as laid down in Diagnostic and Statistical Manual of Mental Disorders (DSM) and International Classification of Diseases (ICD) diagnostic manuals. Issues such as validity, usefulness and acceptability of the diagnoses in this manual are increasingly debated. Several alternative possibilities have been suggested including: (i) the introduction of truly cross-cutting dimensional measures, that would facilitate dynamic multidimensional formulations of psychopathology, (ii) the Research Domain Criteria, that may facilitate biological research but move away from clinical symptoms, (iii) a system of personalized diagnosis based on psychopathology as a network of symptoms and contexts, and (iv) enhanced focus on motor alterations, other than catatonia, as a possible additional informative dimension of diagnosis in psychiatry, particularly as a possible marker of underlying neurodevelopmental alterations. We suggest that novel systems of diagnosis are likely to rely more on continuous monitoring of diagnostically relevant information in daily life, complementing retrospective symptom criteria in DSM and ICD. Patients and their families are likely to benefit from these projects, as novel models of diagnosis based on daily life information may be linked more strongly to treatment needs and prognosis.
\end{abstract}

Key words: Ambulatory assessment, diagnosis, movement disorder, psychopathology.

\section{DSM-5 and ICD-11: business as usual?}

In the light of the recent publication of the fifth edition of the Diagnostic and Statistical Manual of Mental Disorders (DSM-5), there is renewed debate about the relative merit of categorical diagnosis, as laid down in DSM and ICD diagnostic manuals, in psychiatry. Issues such as validity, usefulness and acceptability of the diagnoses in this manual have come to the fore like never before (McGorry \& van Os, 2013).

\section{Expanding diagnostic categories and imbalanced inclusion of risk syndromes}

The most often voiced criticisms are of a rather fundamental nature. The fact that from DSM-I to DSM-5, the number of diagnostic categories has increased from around 100 to over 300 categories, in combination with reliabilities that even in specialized field trials do not exceed kappa's of 0.6 for the majority of

\footnotetext{
* Address for correspondence: Professor J. van Os, Maastricht University Medical Centre, Mastricht, The Netherlands.

(Email: j.vanos@maastrichtuniversity.nl)
}

syndromes (Regier et al. 2013), adds to the impression of arbitrariness of categories. There was concern about the inclusion of risk syndromes in DSM-5, which many considered premature in the light of the available evidence. DSM-5 now contains a major imbalance, as a controversial risk syndrome for dementia (Mild Neurocognitive Disorder) has found its way into the manual, while another controversial risk syndrome for psychotic disorder (Attenuated Psychosis Syndrome) was discarded. Both risk syndromes faced similar inconclusive evidence regarding definition, risk function, validity, reliability and treatability, yet one was included and the other not. The main source of confusion with regard to these risk syndromes is the failure to take into account the fact that patients with early symptoms who have passed many filters along the pathway to care, and end up in specialized outpatient clinics, have much higher probabilities of transition to the full syndrome than people with the same symptoms in the general population. The risk of transition therefore has more to do with selection and referral processes resulting in risk enrichment, rather than the symptomatic syndrome per se. For this reason, the definition of Attenuated Psychosis 
Syndrome, conceptually imperfect as it was, stipulated that individuals had to be help seeking, excluding non-help seeking individuals in the general population with low risk of transition. However, it is unclear how the work group who included Mild Neurocognitive Disorder in DSM-5 weighed the epidemiological evidence that risk of transition from Mild Neurocognitive Disorder to dementia is low for the purpose of diagnosis, and that the risk differs as a function of setting (Mitchell \& Shiri-Feshki, 2009). Indeed, most people with mild cognitive impairment will not progress to dementia even after 10 years of follow-up (Mitchell \& Shiri-Feshki, 2009). In addition, with the advent of the dementia risk syndrome in DSM-5, many individuals and their families may feel compelled to undergo expensive diagnostic procedures only to find that there is no treatment even if risk is considered high.

\section{The pluriform algorithm problem: categories really represent groups of sub-categories}

Although DSM and ICD categories create the impression of relatively precise diagnostic categories, the fundamental structure of each category is that of a pluriform algorithm, involving a number of criteria, typically formulated as "three or more of the following symptoms...". This type of shuffling with many different symptoms creates a range of arbitrary sub-categories within the diagnostic category, formed by all possible combinations of signs and symptoms contained with the algorithm. The result is that diagnostic categories are inherently heterogeneous, representing widely different symptom profiles, which is one of the reasons why they cannot be linked consistently to aetiology, treatment and prognosis. Given that the basic function of diagnosis is to provide information about need for care and prognosis, the pluriform algorithmic nature of DSM and ICD appears to undermine rather than facilitate clinical practice. Similarly, the practice to create widely different combinations of signs, symptoms and behaviours that nonetheless are subsumed under the same diagnostic category is unlikely to advance science, particularly if science is conducted within DSM- and ICD-like silos, as it tends to be.

\section{Patient perspective}

While many patients and their families may experience initial relief when receiving a DSM or ICD label, often this is temporary as many will face a bewildering change in diagnosis, or an expanding list of 'comorbid' diagnoses. In addition, over time, many will learn that the diagnosis does not refer to a natural illness type but an ever-changing convention of arbitrary criteria, which change with each revision of the diagnostic manual, and differ between DSM and ICD. Giving a person a categorical diagnosis of a 'disorder' that does not refer to a disease entity in nature can be considered as an act of stereotyping that may contribute to stigma and exclusion that patients experience. In addition, those receiving mystifying labels like schizophrenia will feel written off when confronted with its stereotypical standing as 'devastating brain disease' in academic psychiatry. Patients experience pressure to conform to the stereotype of a brain-diseased individual, resulting in self-stigma in addition to societal stigma. In this context, 'recovery' becomes the act of a patient constructing his own narrative and overcoming the diagnosis (Boevink, 2006).

\section{Possible improvements}

\section{Cross-cutting dimensions did not make it to DSM-5}

One of the possible areas of innovation that DSM-5 would introduce was the addition of cross-cutting 'dimensions'. For example, symptoms of anxiety and depression are common in many if not most mental disorders, suggesting the use of cross-diagnostic dimensions of anxiety and depression. The addition of cross-cutting dimensions would have created a fully dimensionalized system of diagnosis, recognizing, for example, that expression of psychosis is also common - and clinically highly relevant (Perlis et al. 2011; Wigman et al. 2012, 2013a) - in disorders of anxiety and depression and, vice versa, that negative symptoms are also present in bipolar disorder. The addition of dimensions would have reduced the pervasive but scientifically non-productive view of psychopathology representing a myriad of categorically defined disorders, each with their own causes, diagnostic procedure and treatment. It would have opened the way to examination of affective dysregulation, aberrant salience, fear and perceptual alteration independent of the bias of specific categorical disorders. Unfortunately, it did not prove possible to develop valid methodology for cross-cutting dimensions within the time frame of DSM-5. In the short term, inclusion of cross-cutting dimensions is still a viable and important option, and may be explored in future revisions.

\section{The Research Domain Criteria (RDoC) initiative: phenotypes that can be linked to biology but not to symptoms?}

The view expressed by the NIMH is that progress in the diagnosis and treatment of mental disorders is crucially dependent on linking psychopathology to underlying biological functions. According to this 
view, in its essential form, genetic alterations underlie brain alterations that 'cause' mental disorders (Akil et al. 2010). As research suggests that DSM categories cannot be linked conclusively to underlying biological dysfunctions, let alone diagnostically (Kapur et al. 2012), NIMH has proposed alternative phenotypes that may be closer to the underlying biology that is considered to underlie psychopathology (Cuthbert \& Insel, 2010). The RDoC project aims to study basic dimensions of functioning (such as fear, arousal, reward and working memory) to be studied across multiple units of analysis, from genes to neural circuits to behaviours, cutting across disorders as traditionally defined. While interesting, it is not clear how the chosen phenotypes relate to the symptoms that patients' experience, although the implicit suggestion is that they represent the building blocks of psychopathology. For example, it is unclear how the chosen RDoC phenotypes can be linked to psychotic symptoms such as delusions or hallucinations. Therefore, while RDoC phenotypes may represent better targets for experiments linking brain and behaviour in general, they also represent a move away from the experiences of patients, possibly decreasing their relevance for psychiatry.

\section{Psychopathology as a symptom network}

Novel analyses suggest that diagnostic categories in reality may represent complex fuzzy, cross-level causal sets that share signs and symptoms (Borsboom et al. 2011; Kendler et al. 2011; Kendler, 2012; Bringmann et al. 2013) that develop in stages over time (McGorry \& van Os, 2013; Wigman et al. 2013b). For example, stress may lead to insomnia, leading in turn to low mood, which then may cause paranoia, finally resulting in social withdrawal (Freeman et al. 2012; de Wild-Hartmann et al. 2013). Symptoms thus develop from an initial environmental disturbance, followed by a sequence of causal mental events. In this example, paranoia may in turn fuel depression, and depression insomnia, giving rise to negative feedback loops within a network of symptoms (van Os, 2013). The importance of the network concept resides in the fact that it indicates that diagnosis should not focus only on latent constructs of categories or dimensions, but also on how symptoms impact on each other over time, giving rise to complex symptom circuits (Bringmann et al. 2013). In other words, although clinicians naturally are used to think that the symptoms that patients display are indicators of an underlying disease, categorically or dimensionally defined, in fact there may be no strong evidence of underlying diseases, only dynamic circuits of mutually impacting symptoms (Cramer et al. 2010; Borsboom et al. 2011;
Kendler et al. 2011). Each patient may present with a unique symptom circuit that gradually develops over time (van Os et al. 2013; Wigman et al. 2013b). Of course, network models need not be confined to mental symptoms, given strong links between mental and somatic disorders. For example, a person may develop obesity, which may lead to hypertension, which in turn may result in subclinical cardiovascular infarction, followed by arrhythmias, which subsequently cause panic attacks.

\section{Motor dysfunction: a missing link to make diagnosis neurally informative?}

In addition to the above areas of possible novel avenues for psychiatric diagnosis, it is proposed that motor dysfunctions represent a possibly underrepresented area in psychiatric diagnosis. Although 'mental' signs and symptoms to date have been core in the conceptualization of mental disorders, motor signs also may be relevant for the diagnostic framework, notably in the area of catatonia (Walther \& Strik, 2012). (Neurological) motor signs can be linked more directly to brain alterations. Therefore, it may be argued that motor signs should become more prominent in the psychiatric diagnostic process, facilitating examination of links between brain alterations and mental disorders. However, more work is required to relate 'motor' and 'mental' brain phenotypes in the same diagnostic framework. Basal ganglia disorders represent a natural link between movement disorders on the one hand, and behavioural and mental disorders on the other. Both are likely mediated by specific basal-ganglia-thalamocortical circuits (Graybiel, 2000; Kandel, 2013). The association between motor dysfunction and psychopathology is well established as many movement disorders, including idiopathic dystonia and tics, are associated with increased risk of psychopathology. Furthermore, neurodegenerative disorders that are characterized by movement disorder such as Parkinson's or Huntington's disease have very high rates of psychiatric morbidity, which may be traced to the underlying neural areas that are affected.

As these data point to correlations between motor dysfunction and psychiatric diagnosis, the key question is how 'motor' and 'mental' signs and symptoms may interrelate in the same mental-motor circuit, adding to the diagnostic framework. 'Motor' phenotypes are indicators of neural functions that regulate motor control; 'mental' phenotypes refer to, among others, cognition, incentive salience, affect regulation, sensory function and impulse regulation (Fig. 1). There is some evidence that all these different phenotypes interrelate in a dynamic way, possibly reflecting underlying interacting neural networks. For example, in schizophrenia, 


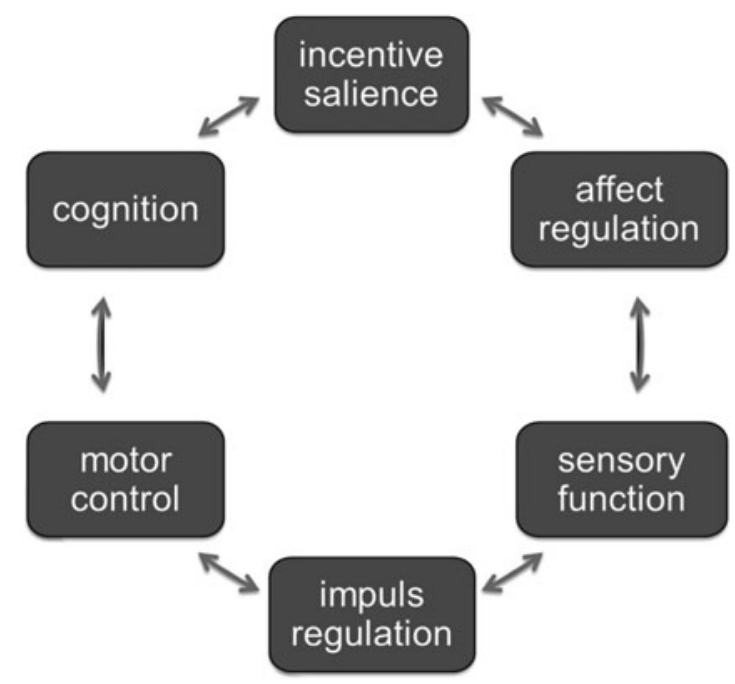

Fig. 1. Networked brain functions linking motor and mental functions. Different brain phenotypes interrelate in a dynamic way, giving rise to an interrelated network of experiences and functions.

there is consistent evidence that dyskinesia is associated with measures of psychopathology (Chakos et al. 1996; Murray \& van Os, 1998; Tenback et al. 2007), and akathisia may exacerbate psychopathology (Duncan et al. 2000). A large body of literature and two meta-analyses (Pappa \& Dazzan, 2009; Koning et al. 2010) show a higher risk of dyskinesia and parkinsonism in drug-naive patients with schizophrenia, and also in first-degree relatives of patients with schizophrenia. Yarden \& Discipio (1971), examining a sample of young and drug-naive patients, showed that abnormal involuntary movements were associated with an early onset and a steadily progressive course, characterized by thought disorder and poor response to medication. In addition, studies by Mittal et al. (Mittal et al. 2007, 2008; Mittal \& Walker, 2007) suggest that spontaneous motor abnormalities are markers of underlying neural alterations, forming part of schizophrenia risk. Studies indicate that orofacial dyskinesia, but not limb-truncal dyskinesia, is associated with cognitive dysfunction and negative symptoms (Waddington et al. 1987; Fenton et al. 1994; Wolff \& O'Driscoll, 1999). Pseudoakathisia also was associated with negative symptoms in one study (Brown \& White, 1991). Both tardive akathisia and tardive dyskinesia may be a marker for negative symptoms as well as for cognitive dysfunction (Sachdev et al. 1996). A prospective study in a sample of 708 patients demonstrated that tardive dyskinesia and negative symptoms developed together (van Os et al. 2000). Findings from another study suggest that orofacial dyskinesia and negative symptoms independently mediate spatial working memory (Pantelis et al. 2001).
Although much of the above work is correlational, and therefore not necessarily diagnostically informative, recent work indicates that motor and cognitive development may be interconnected more closely than previously thought, and that the cerebellum, striatum as well the dorsolateral prefrontal cortex may be relevant to both motor as well as cognitive performance (Diamond, 2000). Indeed, different studies suggest that cognitive functions are associated with drug-induced parkinsonism, for example in elderly patients (Kim et al. 2011), as well as in patients with schizophrenia (Kim et al. 2008; Kim \& Byun, 2009). Studies also demonstrate that (orofacial) tardive dyskinesia and cognitive dysfunctions are associated in schizophrenia (Wegner et al. 1985; Waddington \& Youssef, 1996; Byne et al. 1998; Dodge \& Goldberg, 1999; Krabbendam et al. 2000), as well as in affective disorders (Wolf et al. 1983; Waddington \& Youssef, 1988). In contrast, one study showed superior cued response performance, but equal spatial memory ability, in patients with dyskinesia (Collerton et al. 1985). In addition, findings show that (upper body) movement abnormalities are correlated with neurocognitive deficits in the prodromal period of schizophrenia (Weinberger, 1996; Mittal et al. 2010).

Although the above findings suggest that alterations of the prefrontal cortex play a possible role in tardive dyskinesia, negative symptoms and cognitive dysfunctions (Kirkpatrick \& Buchanan, 1990; Levin, 1984; Goldman-Rakic \& Selemon, 1997; Byne et al. 1998), it is not clear to what degree cognitive dysfunctions (i) are a consequence or a precursor of tardive dyskinesia, (ii) represent an organic vulnerability factor or state marker for TD, or (iii) arise from the same pathophysiologic process as TD (Waddington et al. 1993; Dodge \& Goldberg, 1999).

Nevertheless, the findings (i) corroborate the hypothesis of a common abnormality of basal-ganglia-thalamocortical circuits on the one hand, and indicators of neurodevelopmental liability (cognitive dysfunction, negative symptoms) in schizophrenia and possibly other mental disorders on the other, and (ii) suggest that motor dysfunction reflects an underlying alteration of the dopamine system that also increases risk for schizophrenia. Indeed, subtle dyskinesia was associated with subthreshold psychotic experiences in the general population in a recent study (Mittal et al. 2011). Although the dynamics between motor and mental phenotypes remain poorly understood, the data suggest that motor alterations beyond catatonia are diagnostically relevant, particularly as possible marker of neurodevelopmental alterations. It would be informative if the diagnostic assessment included information on neurodevelopmental liabilities that impact treatment needs and prognosis (Murray, 1994). 


\section{Assessment technologies}

It has been pointed out that collection of diagnostic information in daily life, carried out by patients, has many advantages, creating a collaborative model of a contextually relevant, personal diagnosis that is sensitive to treatment needs and prognosis (van Os et al. 2013). Momentary assessment techniques are available to measure mental symptoms in daily life (Csikszentmihalyi \& Larson, 1987; Delespaul, 1995; Myin-Germeys et al. 2009), while sensor devices to assess motor function are currently being piloted. The advantage of sensor devices is that they are precise and able to measure subtle (subthreshold) motor phenotypes such as bradykinesia, tremor and dyskinesia prospectively in daily life. Therefore, in the near future, it will be possible to measure both mental and motor signs in daily life, possibly providing a diagnostic framework combining the two.

\section{Conclusion}

In conclusion, DSM and ICD categories, while providing a common language for psychiatry, can undermine rather than facilitate clinical practice, and cannot be linked diagnostically to underlying biological dysfunctions (Kapur et al. 2012). Alternative phenotypes as proposed by NIMH, the RDoC phenotypes, may be closer to underlying neurobiology, but also may represent a move away from the experiences of patients, possibly decreasing their relevance for psychiatry. Several novel promising avenues exist to improve psychiatric diagnosis. First, the introduction of cross-cutting dimensions would represent a very helpful short-term improvement of the categorical framework. Second, a system of contextual precision diagnosis based on momentary assessment technology may be a valuable addition, or an alternative, to the diagnostic paradigm. Third, there is evidence that a more enhanced focus on motor dysfunction may be useful for psychiatric diagnosis, particularly as a possible indicator of underlying neurodevelopmental alterations. As motor dysfunction can also be measured with sensor technology in the flow of daily life, it may be combined with psychopathology measures assessed with momentary assessment technology, providing for a more complete diagnostic framework in the near future.

\section{Financial Support}

The project was supported in part by the European Community's Seventh Framework Programme under Grant agreement no. HEALTH-F2-2009-241909 (Project EU-GEI).

\section{Conflict of Interest}

None.

\section{References}

Akil H, Brenner S, Kandel E, Kendler KS, King MC, Scolnick E, Watson JD, Zoghbi HY (2010). Medicine. The future of psychiatric research: genomes and neural circuits. Science 327, 1580-1581.

Boevink WA (2006). From being a disorder to dealing with life: an experiential exploration of the association between trauma and psychosis. Schizophrenia Bulletin 32, 17-19.

Borsboom D, Cramer AO, Schmittmann VD, Epskamp S, Waldorp LJ (2011). The small world of psychopathology. PLoS ONE 6, e27407.

Bringmann LF, Vissers N, Wichers M, Geschwind N, Kuppens P, Peeters F, Borsboom D, Tuerlinckx F (2013). A network approach to psychopathology: new insights into clinical longitudinal data. PLoS ONE 8, e60188.

Brown KW, White T (1991). Pseudoakathisia and negative symptoms in schizophrenic subjects. Acta Psychiatrica Scandinavica 84, 107-109.

Byne W, White L, Parella M, Adams R, Harvey PD, Davis KL (1998). Tardive dyskinesia in a chronically institutionalized population of elderly schizophrenic patients: prevalence and association with cognitive impairment. International Journal of Geriatric Psychiatry 13, 473-479.

Chakos MH, Alvir JM, Woerner MG, Koreen A, Geisler S, Mayerhoff D, Sobel S, Kane JM, Borenstein M, Lieberman JA (1996). Incidence and correlates of tardive dyskinesia in first episode of schizophrenia. Archives of General Psychiatry 53, 313-319.

Collerton D, Fairbairn A, Britton P (1985). Cognitive performance of medicated schizophrenics with tardive dyskinesia. Psychological Medicine 15, 311-315.

Cramer AO, Waldorp LJ, van der Maas HL, Borsboom D (2010). Comorbidity: a network perspective. Behavioral and Brain Sciences 33, 137-150.

Csikszentmihalyi M, Larson R (1987). Validity and reliability of the Experience-Sampling Method. Journal of Nervous and Mental Disease 175, 526-536.

Cuthbert BN, Insel TR (2010). Toward new approaches to psychotic disorders: the NIMH Research Domain Criteria project. Schizophrenia Bulletin 36, 1061-1062.

Delespaul PAEG (1995). Assessing Schizophrenia in Daily Life: the Experience Sampling Method. Maastricht University: Maastricht.

de Wild-Hartmann JA, Wichers M, van Bemmel AL, Derom C, Thiery E, Jacobs N, van Os J, Simons CJ (2013). Day-to-day associations between subjective sleep and affect in regard to future depression in a female population-based sample. British Journal of Psychiatry 202, 407-412.

Diamond A (2000). Close interrelation of motor development and cognitive development and of the cerebellum and prefrontal cortex. Child Development 71, 44-56.

Dodge MR, Goldberg TE (1999). Associations between cognitive impairment and tardive dyskinesia: another perspective. Journal of Clinical Psychiatry 60, 17-21. 
Duncan EJ, Adler LA, Stephanides M, Sanfilipo M, Angrist B (2000). Akathisia and exacerbation of psychopathology: a preliminary report. Clinical Neuropharmacology 23, 169-173.

Fenton WS, Wyatt RJ, McGlashan TH (1994). Risk factors for spontaneous dyskinesia in schizophrenia. Archives of General Psychiatry 51, 643-650.

Freeman D, Stahl D, McManus S, Meltzer H, Brugha T, Wiles N, Bebbington P (2012). Insomnia, worry, anxiety and depression as predictors of the occurrence and persistence of paranoid thinking. Social Psychiatry and Psychiatric Epidemiology 47, 1195-1203.

Goldman-Rakic PS, Selemon LD (1997). Functional and anatomical aspects of prefrontal pathology in schizophrenia. Schizophrenia Bulletin 23, 437-458.

Graybiel AM (2000). The basal ganglia. Current Biology 10, R509-R511.

Kandel ER (2013). Principles of Neural Science, 5th edn. McGraw-Hill Medical: New York.

Kapur S, Phillips AG, Insel TR (2012). Why has it taken so long for biological psychiatry to develop clinical tests and what to do about it? Molecular Psychiatry 17, 1174-1179.

Kendler KS (2012). Levels of explanation in psychiatric and substance use disorders: implications for the development of an etiologically based nosology. Molecular Psychiatry 17, 11-21.

Kendler KS, Zachar P, Craver C (2011). What kinds of things are psychiatric disorders? Psychological Medicine 41, 11431150.

Kim JH, Byun HJ (2009). Non-motor cognitive-perceptual dysfunction associated with drug-induced parkinsonism. Human Psychopharmacology 24, 129-133.

Kim JH, Kim SY, Byun HJ (2008). Subjective cognitive dysfunction associated with drug-induced parkinsonism in schizophrenia. Parkinsonism and Related Disorders 14, 239-242.

Kim YD, Kim JS, Chung SW, Song IU, Yang DW, Hong YJ, Kim YI, Ahn KJ, Kim HT, Lee KS (2011). Cognitive dysfunction in drug induced parkinsonism (DIP). Archives of Gerontology and Geriatrics 53, e222-e226.

Kirkpatrick B, Buchanan RW (1990). The neural basis of the deficit syndrome of schizophrenia. Journal of Nervous and Mental Disease 178, 545-555.

Koning JP, Tenback DE, van Os J, Aleman A, Kahn RS, van Harten PN (2010). Dyskinesia and parkinsonism in antipsychotic-naive patients with schizophrenia, first-degree relatives and healthy controls: a meta-analysis. Schizophrenia Bulletin 36, 723-731.

Krabbendam L, van Harten PN, Picus I, Jolles J (2000). Tardive dyskinesia is associated with impaired retrieval from long-term memory: the Curacao Extrapyramidal Syndromes Study: IV. Schizophrenia Research 42, 41-46.

Levin S (1984). Frontal lobe dysfunctions in schizophrenia-II. Impairments of psychological and brain functions. Journal of Psychiatric Research 18, 57-72.

McGorry P, van Os J (2013). Redeeming diagnosis in psychiatry: timing versus specificity. Lancet 381, 343-345.

Mitchell AJ, Shiri-Feshki M (2009). Rate of progression of mild cognitive impairment to dementia-meta-analysis of 41 robust inception cohort studies. Acta Psychiatrica Scandinavica 119, 252-265.
Mittal VA, Walker EF (2007). Movement abnormalities predict conversion to Axis I psychosis among prodromal adolescents. Journal of Abnormal Psychology 116, 796-803.

Mittal VA, Tessner KD, Trottman HD, Esterberg M, Dhrub SH, Simeonova DI, McMillan AL, Murphy E, Saczawa ME, Walker EF (2007). Movement abnormalities and the progression of prodromal symptomatology in adolescents at risk for psychotic disorders. Journal of Abnormal Psychology 116, 260-267.

Mittal VA, Neumann C, Saczawa M, Walker EF (2008). Longitudinal progression of movement abnormalities in relation to psychotic symptoms in adolescents at high risk of schizophrenia. Archives of General Psychiatry 65, 165-171.

Mittal VA, Walker EF, Bearden CE, Walder D, Trottman H, Daley M, Simone A, Cannon TD (2010). Markers of basal ganglia dysfunction and conversion to psychosis: neurocognitive deficits and dyskinesias in the prodromal period. Biological Psychiatry 68, 93-99.

Mittal VA, Dean DJ, Pelletier A, Caligiuri M (2011). Associations between spontaneous movement abnormalities and psychotic-like experiences in the general population. Schizophrenia Research 132, 194-196.

Murray RM (1994). Neurodevelopmental schizophrenia: the rediscovery of dementia praecox. British Journal of Psychiatry 25, 6-12.

Murray RM, van Os J (1998). Predictors of outcome in schizophrenia. Journal of Clinical Psychopharmacology 18 (Suppl. 1), 2S-4S.

Myin-Germeys I, Oorschot M, Collip D, Lataster J, Delespaul P, van Os J (2009). Experience sampling research in psychopathology: opening the black box of daily life. Psychological Medicine 39, 1533-1547.

Pantelis C, Stuart GW, Nelson HE, Robbins TW, Barnes TR (2001). Spatial working memory deficits in schizophrenia: relationship with tardive dyskinesia and negative symptoms. American Journal of Psychiatry 158, 1276-1285.

Pappa S, Dazzan P (2009). Spontaneous movement disorders in antipsychotic-naive patients with first-episode psychoses: a systematic review. Psychological Medicine 39, 1065-1076.

Perlis RH, Uher R, Ostacher M, Goldberg JF, Trivedi MH, Rush AJ, Fava M (2011). Association between bipolar spectrum features and treatment outcomes in outpatients with major depressive disorder. Archives of General Psychiatry 68, 351-360.

Regier DA, Narrow WE, Clarke DE, Kraemer HC, Kuramoto SJ, Kuhl EA, Kupfer DJ (2013). DSM-5 field trials in the United States and Canada, Part II: test-retest reliability of selected categorical diagnoses. American Journal of Psychiatry 170, 59-70.

Sachdev P, Hume F, Toohey P, Doutney C (1996). Negative symptoms, cognitive dysfunction, tardive akathisia and tardive dyskinesia. Acta Psychiatrica Scandinavica 93, 451-459.

Tenback DE, van Harten PN, Slooff CJ, van Os J (2007). Worsening of psychosis in schizophrenia is longitudinally associated with tardive dyskinesia in the European Schizophrenia Outpatient Health Outcomes study. Comprehensive Psychiatry 48, 436-440. 
van Os J (2013). The dynamics of subthreshold psychopathology: implications for diagnosis and treatment. American Journal of Psychiatry 170, 695-698.

van Os J, Walsh E, van Horn E, Tattan T, Bale R, Thompson SG (2000). Changes in negative symptoms and the risk of tardive dyskinesia: a longitudinal study. UK700 Group. Acta Psychiatrica Scandinavica 101, 300-306.

van Os J, Delespaul P, Wigman J, Myin-Germeys I, Wichers M (2013). Beyond DSM and ICD: introducing "precision diagnosis" for psychiatry using momentary assessment technology. World Psychiatry 12, 113-117.

Waddington JL, Youssef HA (1988). Tardive dyskinesia in bipolar affective disorder: aging, cognitive dysfunction, course of illness, and exposure to neuroleptics and lithium. American Journal of Psychiatry 145, 613-616.

Waddington JL, Youssef HA (1996). Cognitive dysfunction in chronic schizophrenia followed prospectively over 10 years and its longitudinal relationship to the emergence of tardive dyskinesia. Psychological Medicine 26, 681-688.

Waddington JL, Youssef HA, Dolphin C, Kinsella A (1987). Cognitive dysfunction, negative symptoms, and tardive dyskinesia in schizophrenia. Their association in relation to topography of involuntary movements and criterion of their abnormality. Archives of General Psychiatry 44, 907-912.

Waddington JL, O'Callaghan E, Larkin C, Kinsella A (1993). Cognitive dysfunction in schizophrenia: organic vulnerability factor or state marker for tardive dyskinesia? Brain and Cognition 23, 56-70.

Walther S, Strik W (2012). Motor symptoms and schizophrenia. Neuropsychobiology 66, 77-92.
Wegner JT, Kane JM, Weinhold P, Woerner M, Kinon B, Lieberman J (1985). Cognitive impairment in tardive dyskinesia. Psychiatry Research 16, 331-337.

Weinberger DR (1996). On the plausibility of 'the neurodevelopmental hypothesis' of schizophrenia. Neuropsychopharmacology 14 (Suppl. 3), 1S-11S.

Wigman JT, van NM, Vollebergh WA, Lieb R, Beesdo-Baum K, Wittchen HU, van Os J (2012). Evidence that psychotic symptoms are prevalent in disorders of anxiety and depression, impacting on illness onset, risk, and severity-implications for diagnosis and ultra-high risk research. Schizophrenia Bulletin 38, 247-257.

Wigman JT, van Os J, Abidi L, Huibers MJ, Roelofs J, Arntz A, Kelleher I, Peeters FP (2013a). Subclinical psychotic experiences and bipolar spectrum features in depression: association with outcome of psychotherapy. Psychological Medicine, 7, 1-12.

Wigman JT, van Os J, Thiery E, Derom C, Collip D, Jacobs N, Wichers M (2013b). Psychiatric diagnosis revisited: towards a system of staging and profiling combining nomothetic and idiographic parameters of momentary mental states. PLoS One 8, e59559.

Wolf ME, Ryan JJ, Mosnaim AD (1983). Cognitive functions in tardive dyskinesia. Psychological Medicine 13, 671-674.

Wolff AL, O'Driscoll GA (1999). Motor deficits and schizophrenia: the evidence from neuroleptic-naive patients and populations at risk. Journal of Psychiatry and Neuroscience 24, 304-314.

Yarden PE, Discipio WJ (1971). Abnormal movements and prognosis in schizophrenia. American Journal of Psychiatry 128, 317-323. 